\title{
Compatibility between object size and response side in grasping: The left hand prefers smaller objects, the right hand prefers larger objects
}

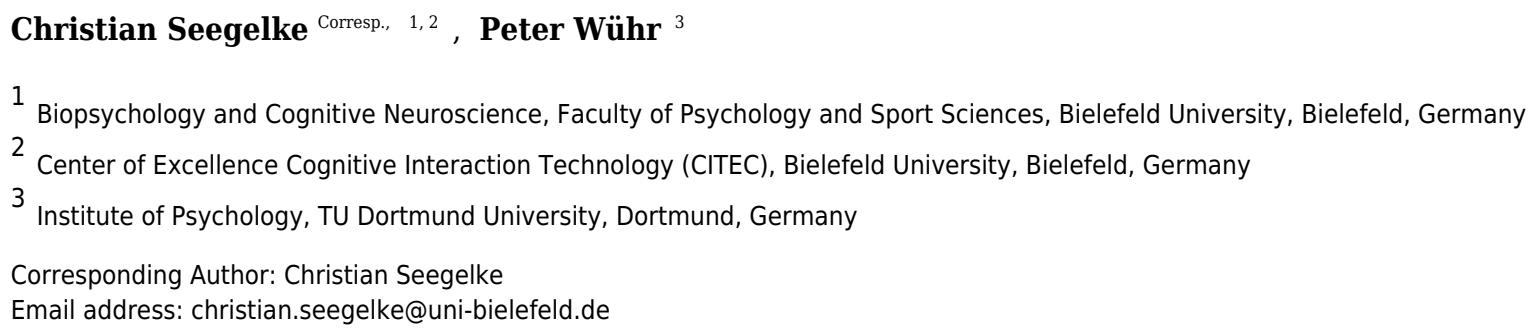

It has been proposed that the brain processes quantities such as space, size, number, and other magnitudes using a common neural metric, and that this common representation system reflects a direct link to motor control, because the integration of spatial, temporal, and other quantity-related information is fundamental for sensorimotor transformation processes. In the present study, we examined compatibility effects between physical stimulus size and spatial (response) location during a sensorimotor task. Participants reached and grasped for a small or large object with either their non-dominant left or their dominant right hand. Our results revealed that participants initiated left hand movements faster when grasping the small cube compared to the large cube, whereas they initiated right hand movements faster when grasping the large cube compared to the small cube. Moreover, the compatibility effect influenced the timing of grip aperture kinematics. These findings indicate that the interaction between object size and response hand affects the planning of grasping movements and supports the notion of a strong link between the cognitive representation of (object) size, spatial (response) parameters, and sensorimotor control. 
1

2 The left hand prefers smaller objects, the right hand prefers larger objects

3

4

5

$6{ }^{1}$ Biopsychology and Cognitive Neuroscience, Faculty of Psychology and Sport Sciences,

7 Bielefeld University, Bielefeld, Germany

$8{ }^{2}$ Center of Excellence Cognitive Interaction Technology (CITEC), Bielefeld, Germany

9 Institute of Psychology, TU Dortmund University, Dortmund, Germany

10

11

12

13

14

15

16

17 Correspondence

18

19

20

21 Universitätsstraße 25

2233615 Bielefeld, Germany

23 E-Mail: $\underline{\text { Christian.Seegelke } @ \text { uni-bielefeld.de }}$

\author{
Christian Seegelke ${ }^{1,2}$, Peter Wühr ${ }^{3}$
}




\section{Abstract}

25 It has been proposed that the brain processes quantities such as space, size, number, and other

26 magnitudes using a common neural metric, and that this common representation system reflects a

27 direct link to motor control, because the integration of spatial, temporal, and other quantity-related

28 information is fundamental for sensorimotor transformation processes. In the present study, we

29 examined compatibility effects between physical stimulus size and spatial (response) location

30 during a sensorimotor task. Participants reached and grasped for a small or large object with either

31 their non-dominant left or their dominant right hand. Our results revealed that participants initiated

32 left hand movements faster when grasping the small cube compared to the large cube, whereas

33 they initiated right hand movements faster when grasping the large cube compared to the small

34 cube. Moreover, the compatibility effect influenced the timing of grip aperture kinematics. These

35 findings indicate that the interaction between object size and response hand affects the planning of

36 grasping movements and supports the notion of a strong link between the cognitive representation

37 of (object) size, spatial (response) parameters, and sensorimotor control.

38

39

40

41

42

43

44

45

46

47

48 


\section{Introduction}

According to "A Theory of Magnitude" (ATOM), there exists a generalized magnitude system in the brain, that processes quantities such as space, size, number, time, and other magnitudes using a common neural metric (Walsh, 2015, 2003). Several neuroscientific studies have provided evidence for such a shared neural representation by showing activation in overlapping areas within the posterior parietal cortex (PPC) during the processing of different magnitudes (e.g., Jacob and Nieder, 2009; Pinel et al., 2004; see Hubbard et al., 2005; Bueti and Walsh, 2009 for reviews). On a behavioral level, this notion is supported by studies showing interaction effects between several dimensions addressed in ATOM such as number and space (Dehaene et al., 1993; Winter et al., 2015), number and size (Henik and Tzelgov, 1982; Reike and Schwarz, 2017)), time and space (Bonato et al., 2012), size and space (Sellaro et al., 2015; Wühr and Seegelke, 2018), and other magnitudes (Macnamara et al., 2018). For example, interactions between numbers and space are evident in the "Spatial-Numerical Association of Response Codes (SNARC)" effect. Here, participants typically respond faster with the left hand in response to (relative) small numbers as compared to (relative) large numbers, whereas they respond faster with the right hand in response to (relative) large numbers as compared to (relative) small numbers (e.g., Dehaene et al., 1993). The SNARC effect has led to the suggestion that numbers are spatially organized along a mental number line (MNL) with small numbers represented to the left and large numbers to the right (Restle, 1970; Dehaene et al., 1993).

According to ATOM, this common representation system evolved through interaction with the environment, as it is through movement that we learn associations between different magnitude domains, for example that larger objects are usually heavier and that it takes more time to cover a larger distance (Walsh, 2003; Binetti et al., 2015). This assumption is corroborated by the fact that 
72 many subregions within the PPC (and particularly in the intraparietal sulcus, IPS) are involved in

73 visuomotor transformations of spatial parameters required for specific motor actions (see

74 Rizzolatti and Luppino, 2001; Gallivan and Culham, 2015 for reviews). Accordingly, this common

75 representation system reflects a functional organization subserving motor control, because the

76 integration of spatial, temporal, and other quantity-related information is fundamental for

77 sensorimotor transformation processes within the PPC (Crawford et al., 2011).

78 The proposal of a strong link between magnitude processing and sensorimotor processes is

79 supported by studies showing direct interactions between magnitude processing and the planning

$80 \mathrm{and} /$ or execution of movements. For example, the SNARC effect is not only expressed in faster

81 reaction times for small-left and large-right associations (e.g., Dehaene et al., 1993), but also

82 evident in movement execution times (Fischer, 2003), and systematic trajectory shifts (Song and

83 Nakayama, 2008) in manual reaching tasks.

84 Besides reaching paradigms, grasping provides a particularly suitable task to study 85 magnitude-motor interactions, since grasping naturally requires the processing of magnitude86 related information. Reach-to-grasp movements exhibit a clear spatio-temporal profile that is 87 characterized by a progressive opening of the grip with its peak (i.e., maximum grip aperture) 88 highly correlated with object size, followed by closing of the grip until it matches the object size 89 (Castiello, 2005). Grasping tasks have often been employed to study interactions between the 90 processing of numerical magnitudes and size-related motor parameters. In an initial study, Andres 91 et al. (2004) showed that participants initiated hand-closing movements faster in response to small 92 numbers and hand-opening movements faster in response to larger numbers. Although the task did 93 not require the grasping of an object, the authors still argued that this interaction arose due to a 94 common magnitude representation for number processing and the computation of an appropriate 
95 grip aperture. Subsequent studies provided more conclusive evidence for this claim (Lindemann

96 et al., 2007; Andres et al., 2008). These studies demonstrated that precision grips are initiated faster

97 in response to small numbers whereas power grips are initiated faster in response to large numbers

98 (Lindemann et al., 2007), and that grasping kinematics exhibit increased grip aperture in the

99 presence of large numbers (Lindemann et al., 2007; Andres et al., 2008; Namdar et al., 2014).

100 Together, these findings strongly suggest that number magnitude processing interacts with the 101 sensorimotor processes involved in shaping the hand grip to object size.

102 Although most studies have examined interactions between numerical information and 103 motor-related spatial coding (e.g., Andres et al., 2004; Andres et al., 2008; Lindemann et al., 2007;

104 Namdar et al., 2014; Namdar and Ganel, 2018; Rugani et al., 2018; Rugani et al., 2017; Badets

105 and Pesenti, 2010; Ranzini et al., 2011), similar interactions have also been observed for other 106 magnitude domains. For example, when participants reached out to grasp a wooden block, their

107 movements had a larger grip aperture after reading a word representing a larger object (e.g. apple) 108 than reading a word representing a small object (e.g., grape, Glover et al., 2004), indicating 109 interactions between (conceptual) object size and reach-to-grasp movements (see also Gentilucci 110 et al., 2000; Glover and Dixon, 2002).

111 In the present study, we address two domains whose potential interactions have largely been 112 neglected in the literature, but clearly are relevant for sensorimotor control: physical object size 113 and space. Recently, we demonstrated a SNARC-like compatibility effect between physical object 114 size and (horizontal) response location using a classic stimulus-response (S-R) compatibility task 115 that required a left or right button press in response to a large or small square in each trial (Wühr 116 and Seegelke, 2018). Specifically, we found that with the left hand, participants responded faster 117 to small stimuli than to large stimuli, whereas with the right hand, they responded faster to large 
118 stimuli than to small stimuli (see Ren et al., 2011 for similar results). This size-space compatibility

119 effect was also observed when stimulus size was irrelevant for task performance, indicating some 120 degree of automaticity in this size-response interaction.

121 With the present study, we aimed at replicating and extending our previous findings. 122 Specifically, and based on the proposition that magnitude processing and motor control share a 123 common representation (Bueti and Walsh, 2009; Walsh, 2003), we examined whether the 124 compatibility effect between physical object size and space (i.e., horizontal response location) 125 would also influence motor-related magnitude processing. To this end, we extended our paradigm 126 to a visually-guided grasping task. Participants reached and grasped for a centrally positioned 127 small or large object (a cube of $8 \mathrm{~cm}^{3}$ or $64 \mathrm{~cm}^{3}$ ) using either their left or right hand.

128 We made the following predictions: First, we expected to replicate the compatibility effect 129 between physical object size and horizontal response location (Ren et al., 2011; Wühr and 130 Seegelke, 2018). That is, reaction times should be shorter for small-left than for large-left 131 associations and shorter for large-right than for small-right associations. Second, given that areas 132 within the PPC are concerned with magnitude processing as well as sensorimotor transformations 133 of spatial parameters required for specific motor actions (Gallivan and Culham, 2015; Bueti and 134 Walsh, 2009), we expected that the compatibility effect should not only be present in reaction 135 times, but also influence kinematic parameters of an action, particularly size-related parameters 136 (i.e., latency and amplitude of maximal grip aperture). Third, in the context of numerical-spatial 137 interactions, it has been reasoned that interaction effects between different magnitudes might be 138 stronger in a visually guided motor task compared to a keypress response task due to the stronger 139 involvement of sensorimotor processing within parietal areas in such a task (Fias et al., 2001). 
140 Consequently, we expected that the compatibility effect should be stronger in the present study

141 compared to our previous study (Wühr and Seegelke, 2018).

142 Methods

143 Participants

144 Based on previous research (Wühr and Seegelke, 2018), we defined a target sample size of

14524 right-handed participants. We collected data from 31 individuals from Bielefeld University

146 because we excluded data from 7 participants (see below). All participants had normal or corrected

147 to normal vision, were physically and neurologically healthy, and received course credit in

148 exchange for their participation. We removed five participants from analysis as they exhibited

149 more than $20 \%$ erroneous trials during either the compatible or incompatible conditions.

150 Furthermore, we removed one participant due to technical problems with kinematic data recording

151 and one left-handed participant. The final sample thus consisted of 24 participants (mean age =

15222.83 years; $\mathrm{SD}=3.37$ years, range $=19-32$ years; 9 female, 15 male; mean handedness score $=$

$15398.41, \mathrm{SD}=5.40$, range $=80-100 ;$ Dragovic, 2004). The study was approved by the Bielefeld

154 University Ethics committee (Ethical Application Ref: 2017-114), and all the participants provided

155 written informed consent in accordance with the Declaration of Helsinki.

156 Apparatus

157 The experimental setup was positioned on a custom-made shelf $(200 \mathrm{~cm} \times 60 \mathrm{~cm})$ at a height 158 of $80 \mathrm{~cm}$. It consisted of two square blocks made of PVC $(10 \times 10 \times 3 \mathrm{~cm})$ with centrally embedded 159 disks ( $7 \mathrm{~cm}$ in diameter) which served as start button for the left and right hand, respectively. The 160 start buttons were located at the front edge of the shelf and spaced $40 \mathrm{~cm}$ apart. Another square 161 PVC block $(18 \times 18 \times 3 \mathrm{~cm})$ with a centrally embedded disk $(14.5 \mathrm{~cm}$ in diameter $)$ was placed 30 $162 \mathrm{~cm}$ and centrally behind the start buttons and served as the object base. The manipulated objects 
163 were two black cubes (small object: 2 × 2 × $2 \mathrm{~cm}$; large object: 4 × 4 × $4 \mathrm{~cm}$ ) and weighed 62 and

$164178 \mathrm{~g}$, respectively. The experimental procedure was controlled via Presentation $\AA$

165 (Neurobehavioral Systems, Berkeley, USA). Participants wore head phones and custom-made

166 visual occlusion glasses which could be rapidly $(<8 \mathrm{~ms})$ made transparent or opaque during the 167 experiment.

168 An optimal motion capture system consisting of 10 Bonita cameras with a spatial precision 169 of approximately 1mm (Vicon Motion Systems, Oxford, UK) was used to record kinematic data 170 at $200 \mathrm{~Hz}$ sampling frequency. Five retro-reflective markers (10mm in diameter) were attached to 171 the distal end of the third metacarpal (MCP), the styloid process of the radius (WRT), the styloid 172 process of the ulna (WRP), the thumb nail (TB), and the index finger nail (IDX) of each hand. The 173 motion capture system was synchronized with Presentation ${ }^{\circledR}$.

174 Procedure

175 After filling out the informed consent and handedness inventory, the retro-reflective markers 176 were placed on each hand. Participants sat centrally in front of the experimental setup at a distance 177 so that they could comfortably reach the object. At the start of each trial, the glasses turned opaque 178 and the experimenter then placed one of the objects at the center of the object base. The 179 experimenter carefully paid attention that the participant was unable to identify the size of the 180 object from the sound of placing it at the object base. After a verbal signal from the experimenter, 181 participants closed their index finger and thumb and depressed the start buttons with the side of 182 their palms. This triggered the presentation of a low tone $(250 \mathrm{~Hz}$, duration $500 \mathrm{~ms})$, informing 183 participants that a trial was initiated. After $1000 \mathrm{~ms}$, the occlusion glasses turned transparent and 184 participants grasped the object from the object base with either the left or the right hand (depending 
185 on condition), lifted the object, and placed it back at the object base. Participants were instructed 186 to perform the task as quickly and accurately as possible.

187 There were four different experimental conditions, resulting from factorial combination of 188 each level of the two factors Response (left hand, right hand) and S-R mapping (compatible, 189 incompatible). For the compatible conditions, participants were required to grasp the small object 190 with the left hand and the large object with the right hand. This mapping was reversed for the 191 incompatible conditions. The factor S-R mapping was blocked, and the order of presentation was 192 counterbalanced across participants. Within each block, participants performed 60 trials (30 with 193 each hand), yielding a total of 120 trials. To familiarize participants with the current mapping, they 194 performed 10 practice trials prior to the first block and 20 practice trials prior to the second block.

\section{Data Processing}

196 We first reconstructed the 3D coordinates of the retro-reflective markers and labeled them 197 manually. We interpolated missing data points using a cubic spline (for gaps $<=10$ frames) or the 198 pattern fill algorithm (for gaps $>10$ frames) in Vicon Nexus 1.8.5, and low-pass filtered the data using a second-order Butterworth filter with a cutoff frequency of $10 \mathrm{~Hz}$. We used custom written MatLab scripts (The MathWorks Version R2015a) for further kinematic post-processing.

We restricted our kinematic analyses to the reach-to-grasp phase, which we defined as the time

202 period between movement initiation and object lift. We calculated the wrist joint center (WJC) of 203 each hand as the midpoint between WRT and WRP. We defined reaction time (RT) as the time 204 between when the glasses turned transparent and movement onset, which we calculated as the time 205 at which the resultant velocity of WJC reached $50 \mathrm{~mm} / \mathrm{s}$. We defined movement time (MT) as the 206 time between movement onset and object lift, as registered by micro switches in the object base. 
207 Finally, we calculated grip aperture of each hand as the Euclidean distance of TB and IDX in 3D 208 space.

209 We excluded trials from analysis in which participants initiated their movements too early $210(0.3 \%)$, too slow $(0.1 \%)$, used the wrong hand $(6.2 \%)$, or in which the $3 \mathrm{D}$ coordinates of the 211 markers could not be reconstructed (1.6\%). In addition, we excluded trials in which RTs were $<$ $212200 \mathrm{~ms}$ or $>750 \mathrm{~ms}(1.5 \%)$ and trials in which MTs were $>1000 \mathrm{~ms}(<0.1 \%)$.

\section{Statistical approach}

214 We performed statistical analyses of the data with a Bayesian model equivalent to a 215 frequentist two-way repeated measures ANOVA using JASP (version 0.8.6; JASP Team, 2018; 216 Wagenmakers et al., 2018a). Bayesian hypotheses testing involves a comparison of at least two 217 different models (e.g., a model assuming a particular main effect and a null model that states the 218 absence of such an effect) and these models need to be fully specified. The prior model odds 219 indicate the relative plausibility of the competing models before seeing the data. The analysis 220 estimates the posterior model odds (i.e., the relative probability of the models after observing the

221

222

223

224 225 226 227 228 data) given the relative predictive probabilities of the models and the prior odds. The emphasis of Bayesian hypothesis testing is on the change in odds from prior to posterior brought about by the data, a quantity referred to as the Bayes factor. Hence, a Bayes factor hypothesis test indicates under which model the observed data are most likely (Wagenmakers, 2007; Wagenmakers et al., 2018b). Lee and Wagenmakers (2013) provide a descriptive and approximate classification scheme of the evidential strength associated with the Bayes factor values $(1=$ no evidence; $1-3=$ anecdotal evidence; 3-10 = moderate evidence; $10-30=$ strong evidence; $30-100=$ very strong evidence; $>100=$ extreme evidence). 
229 Our analyses considered all possible model comparisons, and hence captured all main effects and 230 their interaction of our factorial design [i.e., S-R mapping (compatible, incompatible) and 231 Response hand (left, right)]. Analyses were conducted separately on the following dependent 232 variables: RT, MT, peak velocity, time to peak velocity (in ms), maximal grip aperture, time to 233 maximal grip aperture (in $\mathrm{ms}$ ).

\section{Results}

235 RTs

As shown in Fig. 1, mean RTs were shorter with the compatible than with the incompatible

237

238

239

240

241

242

243

244

245

246

247 248 249

250

251 the interaction term $($ Bayes factor $=19583)$. 

velocity was larger with the compatible mapping than with the incompatible mapping (mean difference $=82 \mathrm{~mm} / \mathrm{s}$ ), whereas for left hand responses peak velocity was larger with the incompatible mapping than with the compatible mapping (mean difference $=56 \mathrm{~mm} / \mathrm{s}$ ). As with MTs, compared to the Null model, the only model that received substantial support from the data was the full model (Bayes factor $=1977$ ). Thus, these findings reflect that reach-to-grasp movements are often faster (i.e., shorter MTs and higher peak velocities) when grasping large as compared to small objects (Seegelke et al., 2016; Castiello et al., 1993). Analysis on time to peak velocity showed that the data were best explained by the Null model or the Compatibility model. The Bayes factor was $1.42(1 / 0.704)$ in favor of the Null model, and hence only indicating anecdotal evidence according to the classification scheme. All other models received considerably less evidence (Bayes factors between 5 and 15 in favor of the Null model).

Maximal grip aperture for right hand responses was larger with the compatible (mean $=102$ $\mathrm{mm})$ than the incompatible mapping $($ mean $=71 \mathrm{~mm})$. In contrast, for left hand responses maximal grip aperture was larger with the incompatible $($ mean $=101 \mathrm{~mm})$ than the compatible mapping $267($ mean $=71 \mathrm{~mm})$, indicating that maximal grip aperture scales with object size (cf. Castiello, 2005). 268 The Bayes factor was $4.300 \mathrm{e}+48$ in favor of the full model compared to the Null model. 269 Furthermore, as shown in Fig. 2, maximal grip aperture was reached, on average, later with the 270 incompatible mapping (388 ms) than with the compatible mapping (367 ms). This difference was 271 more pronounced for the right hand (mean difference $=34 \mathrm{~ms}$ ) than the left hand (mean difference $272=8 \mathrm{~ms}$ ). The analysis showed that both the Compatibility model and the full model received 273 substantial evidence compared to the Null model (Bayes factor $=99.82$ and 66.70, respectively). 
274 The evidence in favor of the Compatibility model over the full model was only a factor of 1.5 $275(99.82 / 66.70)$.

276 In sum the data demonstrate a strong compatibility effect that is evident in both RTs and 277 kinematic parameters (i.e., time to maximal grip aperture).

\section{Discussion}

279 The present study examined size-space interactions during the performance of a visuomotor 280 task. Participants reached and grasped a small or a large cube with either their non-dominant left

281

282 283 284 285 286 287 288 289 290 291 292 293 294 295 296 or their dominant right hand. Our results revealed a compatibility effect between physical object size and horizontal response position (i.e., response hand). Specifically, participants initiated left hand movements faster when grasping the small cube compared to the large cube, whereas they initiated right hand movements faster when grasping the large cube compared to the small cube. These findings thus replicate and extend findings from previous studies (Ren et al., 2011; Wühr and Seegelke, 2018) and demonstrate for the first time that the small-left/ large-right association between physical object size and response position is also present when the physical object size is clearly task-relevant. Furthermore, the compatibility effect also influenced the timing of grip aperture kinematics. On average, maximal grip aperture was reached earlier during compatible compared to incompatible trials. Together, these findings support the idea of a common neural metric underlying magnitude processing and sensorimotor control (Bueti and Walsh, 2009; Walsh, 2003, 2015).

An intriguing and open question which cannot easily be answered by ATOM is the direction of the observed compatibility effect. ATOM assumes some monotonic mapping of quantities, that is, more in one domain should correlate with more in another domain (Walsh, 2015; Bueti and Walsh, 2009). However, this concept cannot be readily applied to the (horizontal) spatial domain 
297 (i.e., left and right). Similarly, in the context of numerical-spatial interactions (e.g., SNARC 298 effect), the origin of the orientation of the mental number line (MNL) remains a debated topic 299 (Rugani and Sartori, 2016).

300 It has been suggested that the MNL originated from several cultural habits such as reading 301 and writing direction (Dehaene et al., 1993; Zebian, 2005; Shaki and Fischer, 2008; Shaki et al., 302 2012; Shaki et al., 2009) or finger counting direction (Fischer, 2008). However, numerical-spatial 303 interactions have also been observed in preverbal infants (Hevia and Spelke, 2010; Hevia et al., 304 2014; Bulf et al., 2016), non-human primates (Adachi, 2014; Drucker and Brannon, 2014), and 305 even birds (Rugani et al., 2015; Rugani et al., 2011). For example, even 3-day old domestic chicks, 306 after being familiarized with a target number, associated a smaller number with left space and a 307 larger number with right space to obtain a food reward (Rugani et al., 2015). Given that the 308 numerical magnitude influenced the chicks' response selection (i.e., go to the left vs. go to the 309 right), it might constitute a link between (numerical) magnitude processing and action (Rugani and 310 Sartori, 2016). These findings suggest that the MNL, rather than being "culturally learned", 311 originated from pre-linguistic and biologically determined precursors, maybe imposed by 312 hemispheric asymmetries related to visuospatial attention (Rugani et al., 2015).

313 Similarly, the compatibility effect between physical object size and response hand observed in the 314 present and previous studies (Ren et al., 2011; Wühr and Seegelke, 2018) might be related to 315 hemispheric lateralization as well, though the sources might be different. There are marked 316 differences in the performance capabilities between the two hands (i.e., manual asymmetries; cf. 317 Goble and Brown, 2008). For example, in right-handers, the dominant hand can produce greater 318 forces than the non-dominant left hand (Petersen et al., 1989; Armstrong and Oldham, 1999; Incel 319 et al., 2002), and this asymmetry is already present in childhood (Hepping et al., 2015). 
320 Consequently, this strength difference could entail a preference to grasp and lift larger (and

321 heavier) objects with the dominant (right) hand than the non-dominant (left) hand.

322 We also found that, besides reaction times, the compatibility effect influenced the timing of

323 grip aperture kinematics. Specifically, maximum grip aperture was reached later during

324 incompatible trials compared to compatible trials for both the right and the left hand. Previous

325 studies that have examined interactions between magnitude processing and sensorimotor control

326 have found that these effects are often evident in movement initiation times (e.g., Lindemann et

327 al., 2007; Badets and Pesenti, 2011; Moretto and Di Pellegrino, 2008) or during the initial stages

328 of the movement trajectory (Andres et al., 2008; Glover et al., 2004; Glover and Dixon, 2002;

329 Namdar et al., 2014). Along with the interpretations from these studies, we propose that the effect

330 of magnitude processing on the timing of grip aperture in the present study reflects an interaction

331 during motor planning stages, which typically occur before movement onset (Wong et al., 2015).

332 However, motor planning is not considered a single and unified building block but is comprised

333 of several sub-stages such as the choice and the description of the motion of the effector and the

334 specification of the motor command (Wong et al., 2015). Hence, it is still unclear at what exact

335 stage the interaction originates - a question on which we will elaborate after the next paragraph.

336 Counter to our expectation, the compatibility effect did not influence maximal grip aperture.

337 A potential reason for this null effect might be that participants adjusted their grip scaling during

338 action execution. Although grip aperture is at least partly determined before movement onset

339 (Jakobson and Goodale, 1991), maximal grip aperture is typically smaller when movements are

340 executed in the presence of vision compared to when online vision is prevented (e.g., Fukui and

341 Inui, 2006; Seegelke et al., 2016). Given that in the present study maximal grip aperture occurred 
342 clearly beyond the time necessary to utilize visual feedback (cf. Elliott et al., 2001), it is possible

343 that participants used visual feedback for online-corrections of their grip scaling.

344 We had further reasoned that the compatibility effect should be stronger in the present study

345 compared to our previous study (Wühr and Seegelke, 2018). This prediction was based on the

346 proposal that (numerical-spatial) interference effects (i.e., SNARC) should be more pronounced

347 during tasks that recruit parietal areas (for example visuomotor tasks) and thus exhibit more neural

348 overlap with magnitude processing (Fias et al., 2001). In the study of Fias et al. (2001), participants

349 were required to respond to stimulus attributes that were more (orientation) or less (shape, color)

350 associated with parietal cortex while ignoring concurrently presented digits. The authors found a

351 SNARC effect (i.e., faster left responses in the presence of task-irrelevant small numbers, faster

352 right responses in presence of task-irrelevant large numbers) for the orientation based judgments 353 only.

354 In another study (Badets et al., 2007), participants judged the graspability of rods of different

355 lengths. Participants overestimated their grasp when the presentation of the rod was preceded by a

356 small number. Conversely, participants underestimated their grasp when a large number preceded

357 the presentation of the rod. In contrast, when participants only compared the length of two

358 successively presented rods (a pure perceptual judgment that does not involve any motor

359 processes), numerical magnitude had no influence on the perceptual size judgment. Together, these

360 results suggest that magnitude-interaction effects are present only (or at least more pronounced)

361 when sensorimotor processes are involved, probably due to the larger extent of overlap in neural

362 substrates in parietal areas.

363 However, counter to that reasoning, the compatibility effect in the present reaching and 364 grasping task (mean RT difference of $27.5 \mathrm{~ms}$ between incompatible and compatible conditions) 
365 was of similar size compared to our previous button press task (30 ms on average; Wühr and

366 Seegelke, 2018). One possible explanation is that the association between physical size and

367 horizontal location originates (mainly) at relatively early motor planning stages that are concerned

368 with effector selection (i.e., using the left vs. the right hand) rather than later stages that are

369 concerned with the specification of movement parameters (Wong et al., 2015). As the decision of

370 what hand to choose was the same in the task of the present study as well as in our previous study

371 (Wühr and Seegelke, 2018), it would seem rather surprising to observe any considerable

372 differences. Of course, this interpretation is rather speculative and it remains certainly possible

373 that task-related differences between the two studies (e.g., with respect to stimuli, task

374 conceptualization, participants, etc.) might have prevented the presence of a stronger effect in the

375 present study.

376 Conclusion

377 In summary, we demonstrated an S-R compatibility effect between stimulus size and 378 response side (i.e. responding hand) in the RTs and in the kinematic parameters of movements for 379 grasping real objects of different size. These findings suggest that the interaction between object 380 size and response hand affects the planning of grasping movements and supports the notion of a 381 strong overlap between the cognitive representation of (object) size and spatial (response) 382 parameters, consistent with ATOM (Walsh, 2003, 2015).

\section{Acknowledgments}

384 We thank Agnieszka Keblowska for her help in data collection and data processing.

\section{References}

Adachi I (2014) Spontaneous spatial mapping of learned sequence in chimpanzees: evidence for a SNARC-like effect. PLoS ONE 9:e90373.

388 Andres M, Davare M, Pesenti M, Olivier E, Seron X (2004) Number magnitude and grip 389 aperture interaction. Neuroreport 15:2773-2777. 
390

391

392

393

394

395

396

397

398

399

400

401

402

403

404

405

406

407

408

409

410

411

412

413

414

415

416

417

418

419

420

421

422

423

424

425

426

427

428

Andres M, Ostry DJ, Nicol F, Paus T (2008) Time course of number magnitude interference during grasping. Cortex; a journal devoted to the study of the nervous system and behavior 44:414-419.

Armstrong CA, Oldham JA (1999) A Comparison of Dominant and Non-Dominant Hand Strengths. Journal of Hand Surgery - British Volume 24:421-425.

Attention and Performance 13.

Badets A, Andres M, Di Luca S, Pesenti M (2007) Number magnitude potentiates action judgements. Experimental Brain Research 180:525-534.

Badets A, Pesenti M (2010) Creating number semantics through finger movement perception. Cognition 115:46-53.

Badets A, Pesenti M (2011) Finger-number interaction: an ideomotor account. Experimental psychology 58:287-292.

Binetti N, Hagura N, Fadipe C, Tomassini A, Walsh V, Bestmann S (2015) Binding space and time through action. Proceedings. Biological sciences 282.

Bonato M, Zorzi M, Umiltà C (2012) When time is space: evidence for a mental time line. Neuroscience and biobehavioral reviews 36:2257-2273.

Bueti D, Walsh V (2009) The parietal cortex and the representation of time, space, number and other magnitudes. Philosophical transactions of the Royal Society of London. Series B, Biological sciences 364:1831-1840.

Bulf H, Hevia MD de, Macchi Cassia V (2016) Small on the left, large on the right: numbers orient visual attention onto space in preverbal infants. Developmental science 19:394-401.

Castiello U (2005) The neuroscience of grasping. Natural Reviews Neuroscience:726-736.

Castiello U, Bennett KMB, Stelmach GE (1993) The bilateral reach to grasp movement. Behavioral Brain Research 56:43-57.

Crawford JD, Henriques DYP, Medendorp WP (2011) Three-dimensional transformations for goal-directed action. Annual review of neuroscience 34:309-331.

Dehaene S, Bossini S, Giraux P (1993) The mental representation of parity and number magnitude. Journal of Experimental Psychology: General 122:371-396.

Dragovic M (2004) Towards an improved measure of the Edinburgh Handedness Inventory: a one-factor congeneric measurement model using confirmatory factor analysis. Laterality 9:411-419.

Drucker CB, Brannon EM (2014) Rhesus monkeys (Macaca mulatta) map number onto space. Cognition 132:57-67.

Elliott D, Helsen WF, Chua R (2001) A century later: Woodworth's (1899) two-component model of goal-directed aiming. Psychological Bulletin 127:342-357.

Fias W, Lauwereyns J, Lammertyn J (2001) Irrelevant digits affect feature-based attention depending on the overlap of neural circuits. Cognitive Brain Research 12:415-423.

Fischer M (2003) Spatial representations in number processing--evidence from a pointing task. Visual Cognition 10:493-508. 
429 Fischer MH (2008) Finger counting habits modulate spatial-numerical associations. Cortex; a 430 journal devoted to the study of the nervous system and behavior 44:386-392.

431 Fukui T, Inui $\mathrm{T}$ (2006) The effect of viewing the moving limb and target object during the early 432 phase of movement on the online control of grasping. Human Movement Science 25:349$433 \quad 371$.

434 Gallivan JP, Culham JC (2015) Neural coding within human brain areas involved in actions.

435 Current Opinion in Neurobiology 33:141-149.

436 Gentilucci M, Benuzzi F, Bertolani L, Daprati E, Gangitano M (2000) Recognising a hand by 437 grasp. Cognitive Brain Research 9:125-135.

438 Glover S, Dixon P (2002) Semantics affect the planning but not control of grasping.

439 Experimental Brain Research 146:383-387.

440 Glover S, Rosenbaum DA, Graham J, Dixon P (2004) Grasping the meaning of words.

441 Experimental Brain Research 154:103-108.

442 Goble DJ, Brown SH (2008) The biological and behavioral basis of upper limb asymmetries in

443 sensorimotor performance. Neuroscience \& Biobehavioral Reviews 32:598-610.

444

445

446

447

448

449

450

451

452

453

454

455

456

457

458

459

460

461

462

463

464

465

466

467

468

Henik A, Tzelgov J (1982) Is three greater than five: The relation between physical and semantic size in comparison tasks. Memory \& Cognition 10:389-395.

Hepping AM, Ploegmakers JJW, Geertzen JHB, Bulstra SK, Stevens M (2015) The Influence of Hand Preference on Grip Strength in Children and Adolescents; A Cross-Sectional Study of 2284 Children and Adolescents. PLoS ONE 10:e0143476.

Hevia MD de, Girelli L, Addabbo M, Macchi Cassia V (2014) Human infants' preference for left-to-right oriented increasing numerical sequences. PLoS ONE 9:e96412.

Hevia MD de, Spelke ES (2010) Number-space mapping in human infants. Psychological Science 21:653-660.

Hubbard EM, Piazza M, Pinel P, Dehaene S (2005) Interactions between number and space in parietal cortex. Nature Reviews. Neuroscience 6:435-448.

Incel NA, Ceceli E, Durukan PB, Erdem H. R., Yorgancioglu ZR (2002) Grip Strength: Effect of Hand Dominance. Singapore Medical Journal 43:234-237.

Jacob SN, Nieder A (2009) Tuning to non-symbolic proportions in the human frontoparietal cortex. The European journal of neuroscience 30:1432-1442.

Jakobson LS, Goodale MA (1991) Factors affecting higher-order movement planning: a kinematic analysis of human prehension. Experimental Brain Research 86:199-208.

JASP Team (2018) JASP.

Lee M, Wagenmakers E-J (2013) Bayesian cognitive modeling: A practical course. New York: Cambridge University Press.

Lindemann O, Abolafia JM, Girardi G, Bekkering H (2007) Getting a grip on numbers: numerical magnitude priming in object grasping. Journal of experimental psychology. Human perception and performance 33:1400-1409.

Macnamara A, Keage HAD, Loetscher T (2018) Mapping of non-numerical domains on space: a systematic review and meta-analysis. Experimental Brain Research 236:335-346. 
469

470

471

472

473

474

475

476

477

478

479

480

481

482

483

484

485

486

487

488

489

490

491

492

493

494

495

496

497

498

499

500

501

502

503

504

505

506

507

508

Moretto G, Di Pellegrino G (2008) Grasping numbers. Experimental Brain Research 188:505515.

Namdar G, Ganel T (2018) Numerical magnitude affects online execution, and not planning of visuomotor control. Psychological Research 82:488-495.

Namdar G, Tzelgov J, Algom D, Ganel T (2014) Grasping numbers: evidence for automatic influence of numerical magnitude on grip aperture. Psychonomic Bulletin \& Review 21:830 835.

Petersen P, Petrick M, Connor H, Conklin D (1989) Grip strength and hand dominance: challenging the 10\% rule. American Journal of Occupational Therapy 43:444-447.

Pinel P, Piazza M, Le Bihan D, Dehaene S (2004) Distributed and Overlapping Cerebral Representations of Number, Size, and Luminance during Comparative Judgments. Neuron 41:983-993.

Ranzini M, Lugli L, Anelli F, Carbone R, Nicoletti R, Borghi AM (2011) Graspable objects shape number processing. Frontiers in Human Neuroscience 5:147.

Reike D, Schwarz W (2017) Exploring the origin of the number-size congruency effect: Sensitivity or response bias? Attention, perception \& psychophysics 79:383-388.

Ren P, Nicholls MER, Ma Y, Chen L (2011) Size matters: Non-numerical magnitude affects the spatial coding of responses. PLOS ONE 6:e23553.

Restle F (1970) Speed of adding and compating numbers. Journal of Experimental Psychology $83: 274-278$.

Rizzolatti G, Luppino G (2001) The Cortical Motor System. Neuron 31:889-901.

Rugani R, Betti S, Ceccarini F, Sartori L (2017) Act on Numbers: Numerical Magnitude Influences Selection and Kinematics of Finger Movement. Frontiers in Psychology 8:1481.

Rugani R, Betti S, Sartori L (2018) Numerical Affordance Influences Action Execution: A Kinematic Study of Finger Movement. Frontiers in Psychology 9:637.

Rugani R, Sartori L (2016) Numbers in Action. Frontiers in human neuroscience 10:388.

Rugani R, Vallortigara G, Priftis K, Regolin L (2015) Number-space mapping in the newborn chick resembles humans' mental number line. Science (New York, N.Y.) 347:534-536.

Rugani R, Vallortigara G, Vallini B, Regolin L (2011) Asymmetrical number-space mapping in the avian brain. Neurobiology of learning and memory 95:231-238.

Seegelke C, Güldenpenning I, Dettling J, Schack T (2016) Visuomotor priming of action preparation and motor programming is similar in visually guided and memory-guided actions. Neuropsychologia 91:1-8.

Sellaro R, Treccani B, Job R, Cubelli R (2015) Spatial coding of object typical size: evidence for a SNARC-like effect. Psychological Research 79:950-962.

Shaki S, Fischer MH (2008) Reading space into numbers: a cross-linguistic comparison of the SNARC effect. Cognition 108:590-599.

Shaki S, Fischer MH, Göbel SM (2012) Direction counts: a comparative study of spatially directional counting biases in cultures with different reading directions. Journal of experimental child psychology 112:275-281. 
509 Shaki S, Fischer MH, Petrusic WM (2009) Reading habits for both words and numbers

510

511

512

513

514

515

516

517

518

519

520

521

522

523

524

525

526

527

528

529

530

531

532

533

534

535

536

537 contribute to the SNARC effect. Psychonomic Bulletin \& Review 16:328-331.

Song J-H, Nakayama K (2008) Numeric comparison in a visually-guided manual reaching task. Cognition 106:994-1003.

Wagenmakers E-J (2007) A practical solution to the pervasive problems ofp values. Psychonomic Bulletin \& Review 14:779-804.

Wagenmakers E-J, Love J, Marsman M, Jamil T, Ly A, Verhagen J, Selker R, Gronau QF, Dropmann D, Boutin B, Meerhoff F, Knight P, Raj A, van Kesteren E-J, van Doorn J, Smira M, Epskamp S, Etz A, Matzke D, de Jong T, van den Bergh D, Sarafoglou A, Steingroever H, Derks K, Rouder JN, Morey RD (2018a) Bayesian inference for psychology. Part II: Example applications with JASP. Psychonomic Bulletin \& Review 25:58-76.

Wagenmakers E-J, Marsman M, Jamil T, Ly A, Verhagen J, Love J, Selker R, Gronau QF, Šmíra M, Epskamp S, Matzke D, Rouder JN, Morey RD (2018b) Bayesian inference for psychology. Part I: Theoretical advantages and practical ramifications. Psychonomic Bulletin \& Review 25:35-57.

Walsh V (2003) A theory of magnitude: common cortical metrics of time, space and quantity. Trends in Cognitive Sciences 7:483-488.

Walsh V (2015) A Theory of Magnitude: the parts that sum to number. In: The Oxford Handbook of Numerical Cognition (Kadosh RC, Dowker A, eds), pp 552-565. New York: Oxford University Press.

Winter B, Matlock T, Shaki S, Fischer MH (2015) Mental number space in three dimensions. Neuroscience \& Biobehavioral Reviews 57:209-219.

Wong AL, Haith AM, Krakauer JW (2015) Motor Planning. The Neuroscientist 21:385-398. Wühr P, Seegelke C (2018) Compatibility between Physical Stimulus Size and Left-right Responses: Small is Left and Large is Right. Journal of Cognition 1:17.

Zebian S (2005) Linkages between Number Concepts, Spatial Thinking, and Directionality of Writing: The SNARC Effect and the REVERSE SNARC Effect in English and Arabic Monoliterates, Biliterates, and Illiterate Arabic Speakers. Journal of Cognition and Culture 5:165-190. 
Figure 1

Effect of compatibility on reaction times

Group mean RTs (large dots) and individual mean RTs (small dots) as a function of S-R Compatibility and Response hand (A). RT difference between the incompatible (IC) and compatible (C) mapping separately for each hand (B). Error bars reflect $95 \%$ credible intervals.
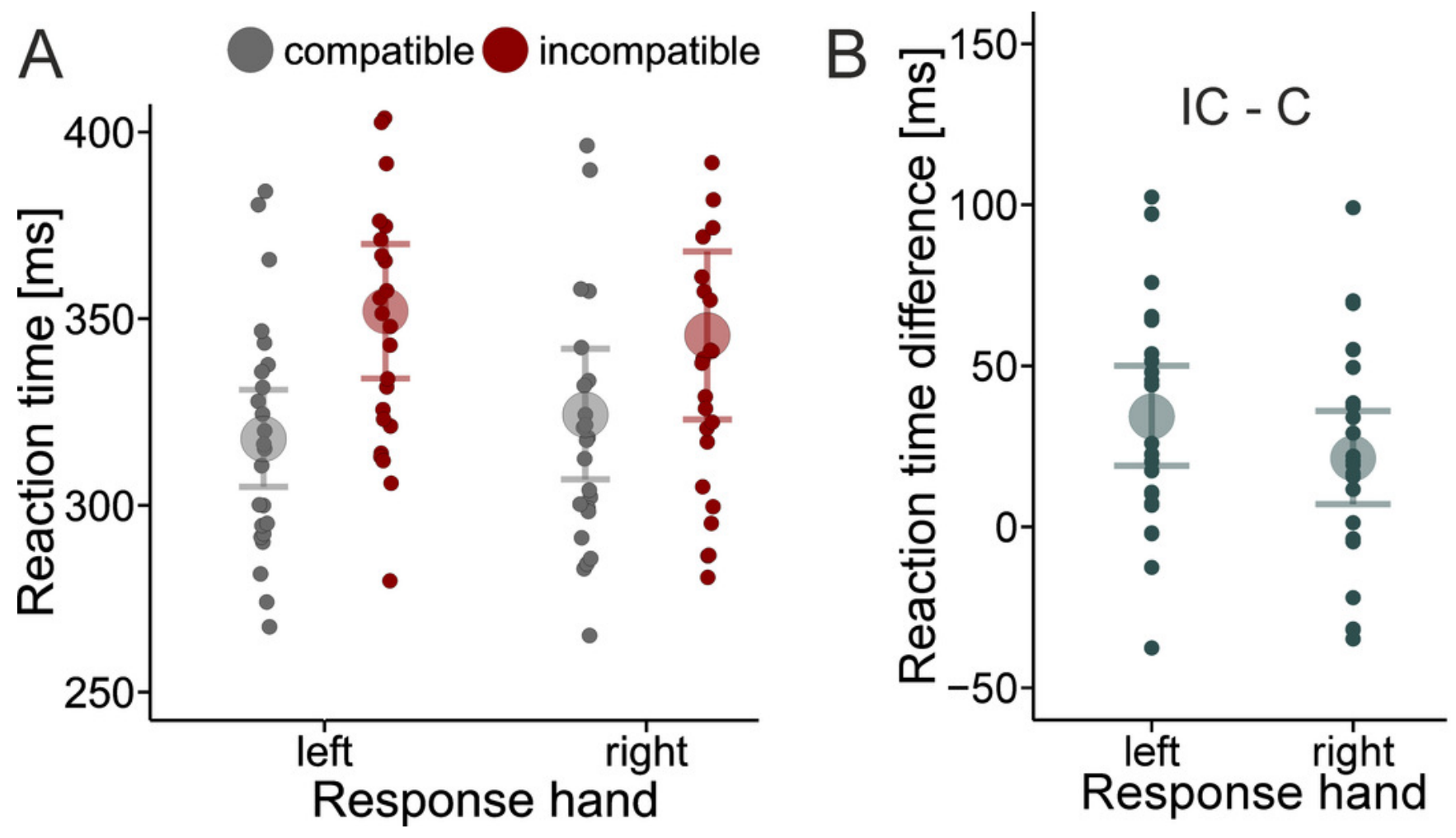
Figure 2

Effect of compatibility on the timing of grip aperture kinematics

Group mean time to maximal grip aperture (large dots) and individual mean time to maximal grip aperture (small dots) as a function of S-R Compatibility and Response hand (A). Time to maximal grip aperture difference between the incompatible (IC) and compatible (C) mapping separately for each hand (B).Error bars reflect 95\% credible intervals.
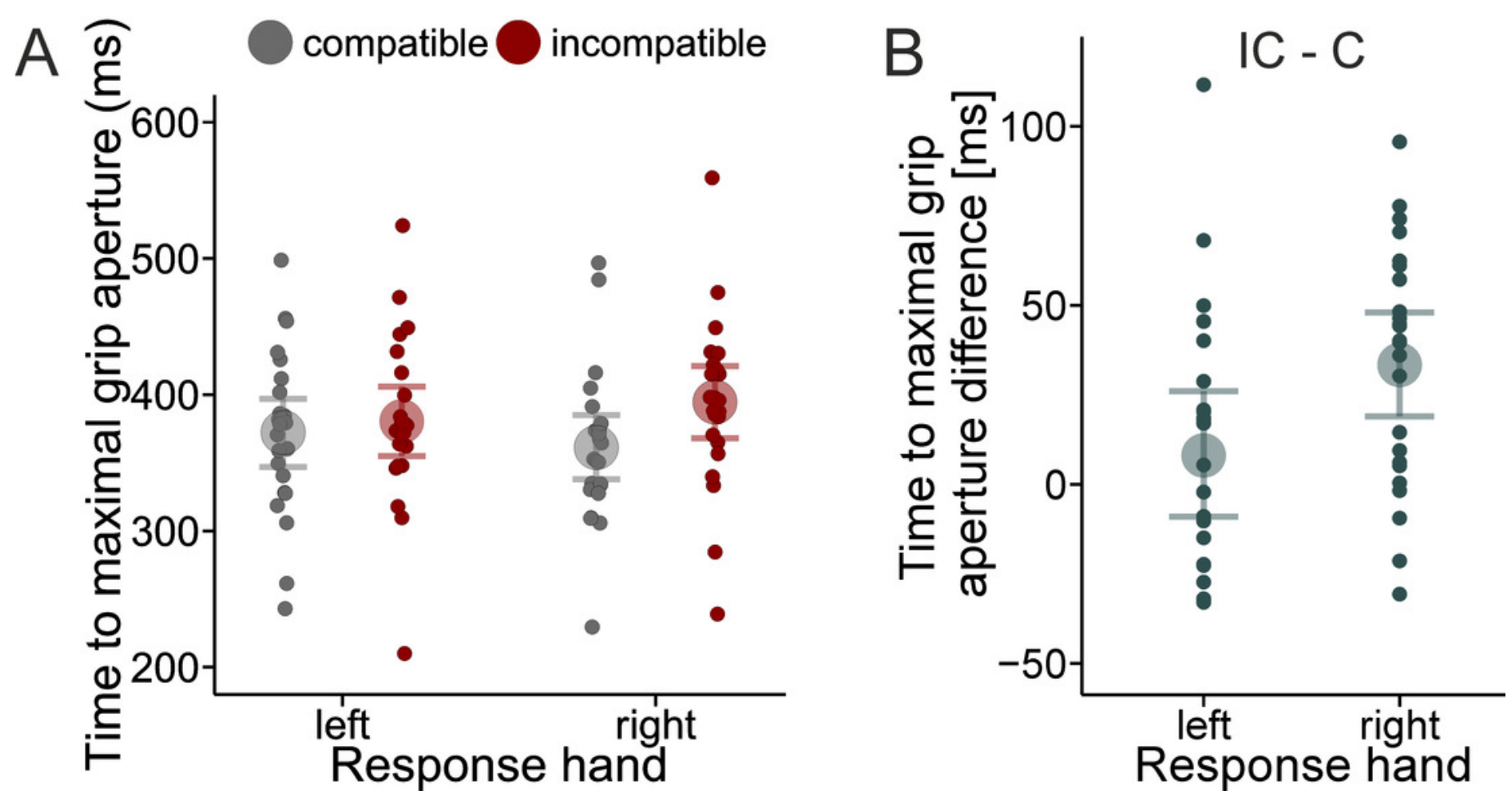